\title{
Integrated Vehicle Thermal Management in Modelica: Overview and Applications
}

\author{
John Batteh ${ }^{1}$ \\ Jesse Gohl ${ }^{1}$ \\ ${ }^{1}$ Modelon, Inc. \\ Ann Arbor, MI \\ United States \\ Sureshkumar Chandrasekar ${ }^{2}$ \\ ${ }^{2}$ Modelon, Inc. \\ Hartford, CT \\ United States \\ john.batteh@modelon.com jesse.gohl@modelon.com \\ chandrasekar.sureshkumar@modelon.com
}

\begin{abstract}
This paper highlights the use of a coordinated suite of Modelica libraries for vehicle thermal management applications. The models are implemented using the Vehicle Dynamics Library, Liquid Cooling Library, and Heat Exchanger Library from Modelon. An integrated vehicle thermal management model is implemented, including the key physical and controls models. The model is used to highlight complex, multi-domain interactions between the physical and control systems over drive cycles for combined thermal and fuel efficiency studies. The model is also used to support controller development and optimization as an FMU integrated into Simulink. The flexibility of FMI-based workflows is also illustrated via batch and Monte Carlo simulations in Excel. A heat exchanger application coupling inputs from CFD illustrates the use of higher fidelity models from Heat Exchanger Library for calculation of performance degradation due to non-uniformity.
\end{abstract}

Keywords: vehicle thermal management; thermal systems; fluid systems; vehicle modeling; powertrain; engine; transmission; controls; Simulink; FMI

\section{Introduction}

To meet increasingly stringent fuel economy and emissions standards, automotive original equipment manufacturers (OEMs) and suppliers have sought novel technologies to increase fuel efficiency. Given the complexity of vehicle systems, the need for increasingly sophisticated analytic tools to perform concept evaluation, capture multi-domain system interactions, and develop and validate control strategies grows. A Modelica-based platform for simulation of vehicle systems is ideal for this type of work as it naturally captures multi-domain interactions, allows flexibility in model complexity to support a range of applications, and supports integration of physical and control systems.

Traditional vehicle models for fuel consumption have often ignored thermal effects as the inclusion of thermal effects increases the model development and parameterization effort and can affect computational efficiency for models which are run on drive cycles which can be thousands of seconds in duration. However, in the search for fuel efficiency gains, advanced technology is rapidly advancing from research and development to production, including the associated controls development. Due to the complex interactions between vehicle subsystems, vehicle thermal management (VTM) requires a holistic approach to minimize energy consumption without violating thermal limits for the various systems. Previous work in Modelica has highlighted this need for thermal management of electrified vehicles [1].

A real world example of a vehicle level technology that has the potential for fuel economy gains, but with a direct impact on thermal management is grill shutters. Grill shutters can be used to restrict or completely close airflow to the front of the vehicle. While this reduction in airflow positively impacts fuel efficiency by reduction in aerodynamic drag, reduced airflow through the front end of the vehicle degrades cooling capacity through the heat exchanger stack which contains the radiator, condenser, and potentially additional coolers such as oil and charge air coolers. Grill shutters can be mechanical or potentially even actively controlled. Clearly attribute balancing at the vehicle level is required to manage fuel consumption gains and the cooling requirements for the various fluid systems over a range of driving conditions and ambient environments. Active grill shutters can be found in production on a range of vehicles from various manufacturers, including the Ford Focus, Chevrolet Cruze, Cadillac ATS, Dodge Dart, and Ram 1500 [1].

To illustrate the complex interactions between vehicle subsystems, vehicle controls, and thermal 
controls, an integrated vehicle thermal management model is implemented with grill shutters. The models are implemented using a coordinated suite of Modelica libraries from Modelon. The models are implemented using the Vehicle Dynamics Library (VDL) [3], Liquid Cooling Library (LCL) [4], and Heat Exchanger Library (HXL) [5]. The model captures vehicle dynamics on drive cycles including key thermal dynamics. The model also includes critical vehicle controls and thermal controls for the grill shutters and fan. This paper provides an overview of the model, key subsystem implementations, and key features of the various libraries to support this type of modeling.

The integrated vehicle thermal management model is used as a demonstrator for Modelica-based workflows for illustrative controls development and robustness applications. The controls development application illustrates the implementation and optimization of an active grill shutter strategy. The Modelica VTM model is exported as an FMU via Functional Mock-up Interface [6] for integration in Simulink with the grill shutter and fan controls. Integration in Simulink is via FMI Toolbox for MATLAB [7] from Modelon. DOE techniques are used to optimize the combined grill shutter and fan settings for minimum fuel consumption. A sample optimized controller is implemented to show the fuel economy impact on several different drive cycles. Sample robustness studies are also performed with the VTM FMU in Excel via FMI Add-in for Excel [8] from Modelon. These applications include batch simulations and Monte Carlo simulations and also illustrate some of the scripting capabilities in FMI Add-in for Excel. Workflows that involve coupling higher fidelity models from Heat Exchanger Library with CFD input data for heat exchanger performance are shown.

This paper describes a coordinated suite of libraries for vehicle thermal management, an integrated model including grill shutters, and applications of this model for controls development and robustness using FMI. These examples illustrate the multidomain approach needed for vehicle thermal management applications. Given the importance of model deployment outside of traditional CAE environments to support model-based systems engineering, workflow aspects via FMI are highlighted.

\section{Integrated VTM Model}

This section outlines the key multi-domain component and subsystem models in the integrated VTM model that support the subsequent applications. The main model components and subsystems are detailed. The model shown is representative of system level models for use in thermal system design and performance characterization with parameterization as a demonstrator model and thus does not include any customer-proprietary data (future publications will highlight industrial models and applications pending publication approval). The vehicle parameterization data was taken from a sedan implemented in VDL. The majority of the thermal parameters were estimated from authors' experience with similar systems in industrial applications.

\subsection{System Model}

Figure 1 shows the integrated VTM model. This model is structured in a thermal-centric way such that the key thermal subsystems are visible at the top level of the model and in parallel with the vehicle model. At this level, the model contains the following subcomponents with key systems to be described in more detail in subsequent sections:

- Lumped 1D conventional vehicle model with automatic transmission

- Lumped thermal models for engine and transmission

- Simple underhood models for engine and transmission heat transfer

- Controllers for fan and grill shutters

- Coolant and transmission oil fluid circuits

- Heat exchanger stack with radiator, condenser, transmission oil cooler, and charge air cooler

- Minimal HVAC and charge air circuits

- Electric fan with simplified vehicle electrical system

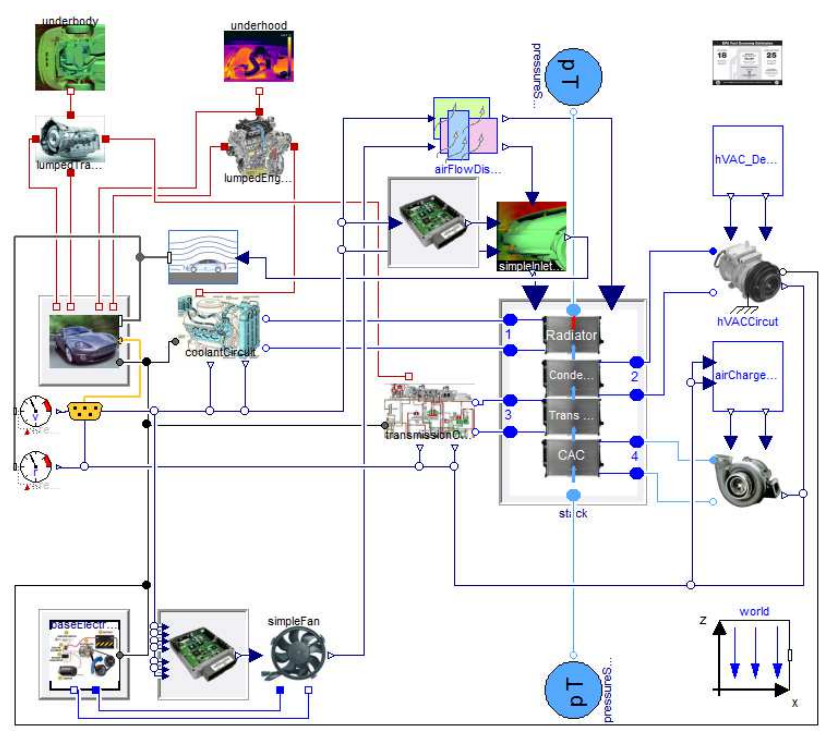

Figure 1. Integrated VTM model 


\subsection{Vehicle Model}

The vehicle model is implemented using the vehicle model architecture and components from Vehicle Dynamics Library [3]. This architecture supports the full range of vehicle models from lumped 1D to geometry-based full 3D representations suitable for vehicle dynamics and handling applications. Previous work [9] has illustrated drivability applications using Vehicle Dynamics Library with simplified chassis representations. A similar approach is used to create a computationally-efficient lumped $1 \mathrm{D}$ vehicle model suitable for drive cycle simulations. This model focuses on core loads and losses to ensure that the engine operates at the appropriate operating conditions for accurate fuel consumption and heat generation. Both the mapped engine and transmission generate heat input to the thermal system via thermal connectors added to the vehicle architecture. The simplified chassis model with rigid axle, no slip tires, and a single lumped mass is shown in Figure 3. Parameterization for the vehicle model is taken from a sedan model in VDL.

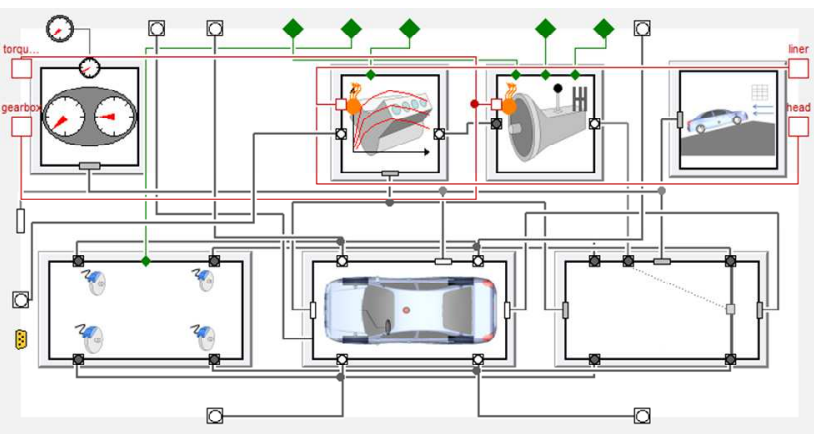

Figure 2. Vehicle model augmented with thermal behavior

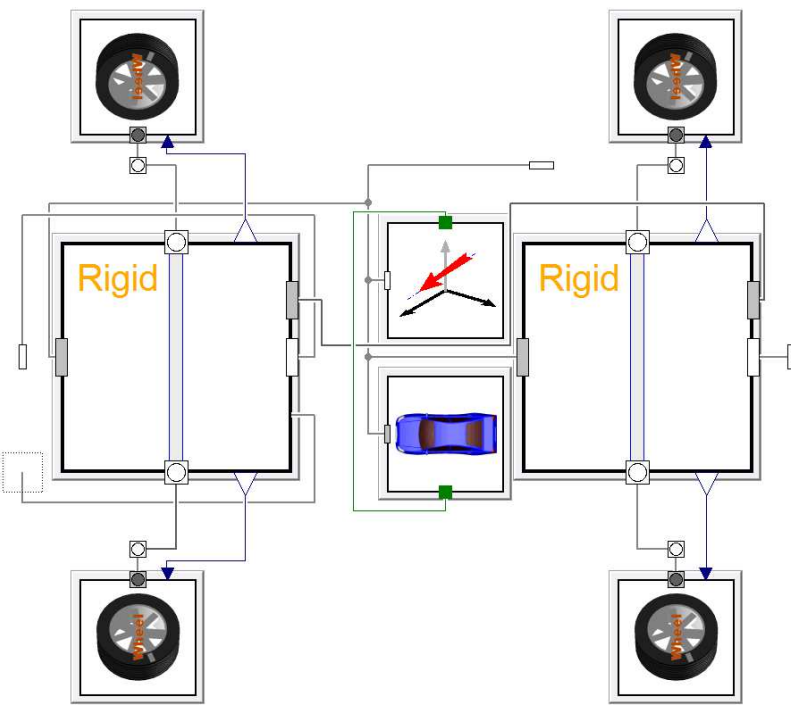

Figure 3. Simplified 1D chassis model

\subsection{Fluid Circuits}

Fluid circuit modeling is a key part of the VTM model. An efficient thermo-fluid implementation is critical as it is common for a VTM model to include several different fluid circuits which interact with the thermal system and heat exchanger stack. Liquid Cooling Library [4] is ideal for modeling incompressible fluid circuits due to its efficient formulations which can support models with minimal number of pressure states, potentially even a single pressure state per circuit, while maintaining thermal states as required throughout the system. This approach eliminates the stiffness and resulting computational impact of modeling incompressible flow circuits as minimally compressible.

Figure 4 shows a simple coolant circuit implemented to support the demonstrator VTM model. This model includes the following components from Liquid Cooling Library:

- Coolant pump driven by crankshaft

- Coolant path through engine head and block

- Thermostat to control flow to radiator and via bypass

- Expansion volume

A similar model is implemented for the transmission oil circuit.

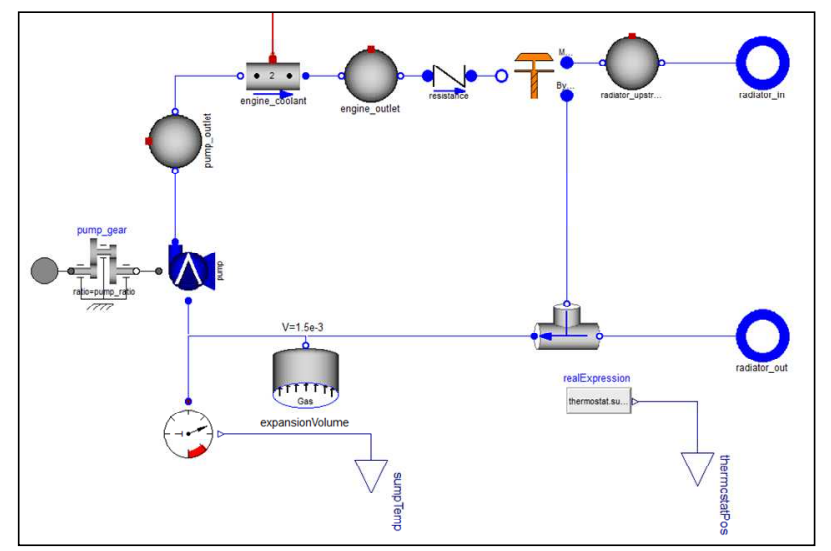

Figure 4. Simple coolant circuit

\subsection{Heat Exchanger Stack}

The heat exchanger stack is a key coupling point between the fluid circuits. Both Liquid Cooling Library and Heat Exchanger Library include models of heat exchanger stacks. These models differ in terms of model detail. Heat Exchanger Library allows detailed, geometry-based models which can be stacked using a streamtube approach for the airflow that preserves non-uniform conditions for each heat exchanger throughout the stack. The heat exchanger models in Heat Exchanger Library can be discretized 
and can accept both uniform and non-uniform airflow inputs. The approach and a sample model are shown in Figure 5. This formulation is efficient enough to support drive cycle work assuming a reasonable number of stream tubes. The stack models in Liquid Cooling Library require only the basic stack geometry and can use mapped heat exchangers with imposed airflow based on external inputs from CFD, etc. The air temperatures through the stack are calculated based on the stack geometry with a single calculated input temperature for each heat exchanger based on preceding heat exchanger outlet temperatures. LCL includes assembled stack models ranging from 2-8 heat exchangers.

Figure 6 shows the heat exchanger stack used in the VTM model. The stack consists of a radiator, condenser, transmission oil cooler, and charge air cooler. Figure 6 shows the visualization of the stack geometry which also provides dynamic visualization of the temperatures during the run. The dynamic summary visualization for the temperatures, flowrates, and heat transfer in each heat exchanger is also shown. Each heat exchanger is implemented as a mapped effectiveness as a table of the fluid and air mass flow rates. Though simplified, effectiveness data is often available early in vehicle programs and thus can support upfront cooling pack concept assessment and early thermal system design and performance.
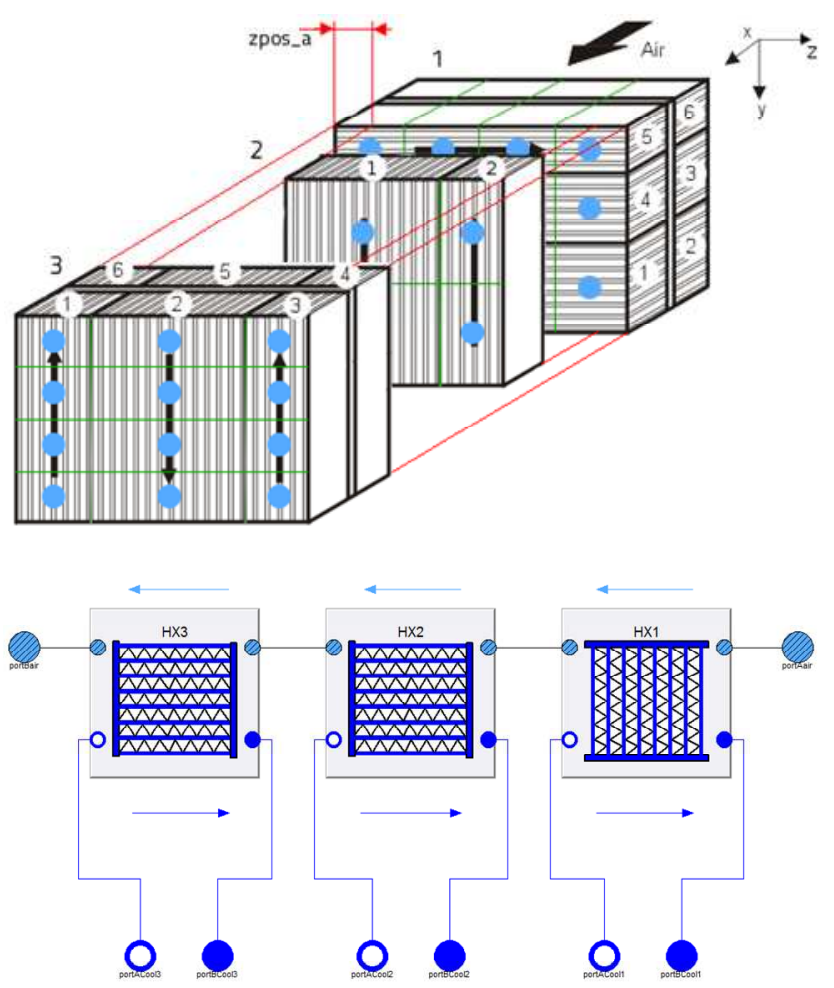

Figure 5. Streamtube approach and sample stack from Heat Exchanger Library

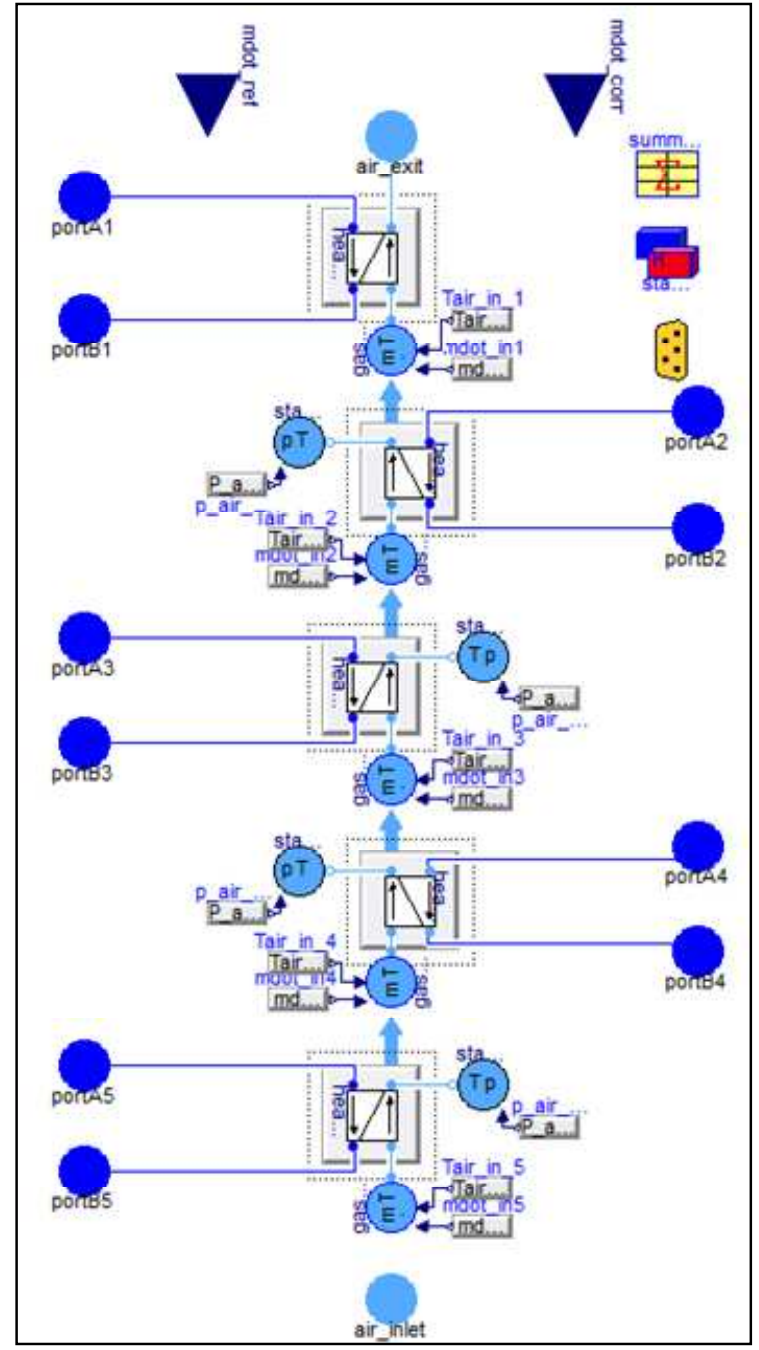

Figure 6. Heat exchanger stack
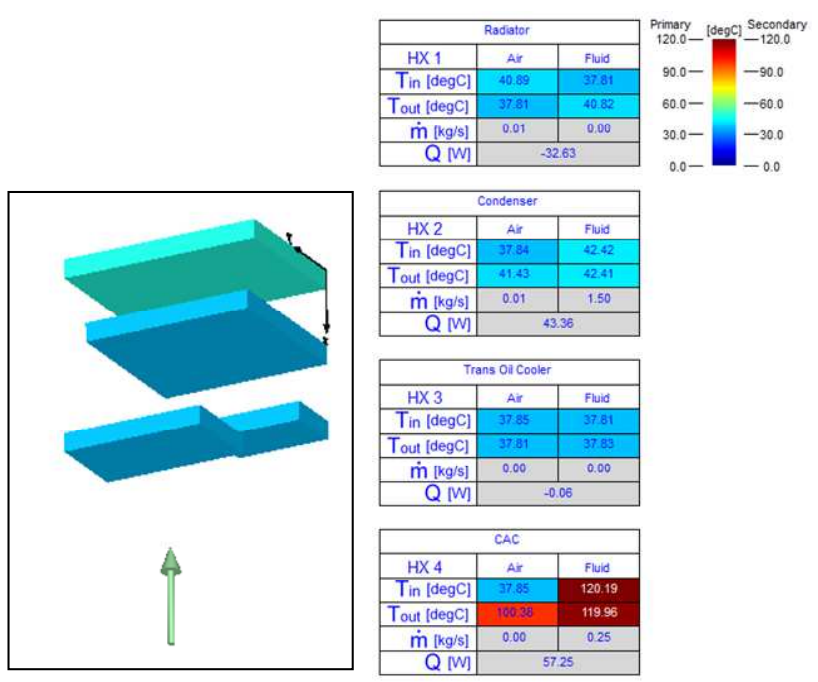

Figure 7. Stack geometry and summary visualization

\subsection{Controllers}

For demonstration purposes, simple controllers in Modelica are implemented for the fan and grill shut- 
ters. The fan controller is shown in Figure 8. The grill shutter implementation mimics a passive grill shutter system where grill shutters are closed above a parameterized vehicle speed as shown in Figure 9. More detailed controller implementations in Simulink are discussed in Section 3.2 when the VTM model is exported as an FMU and combined with the control system in Simulink.

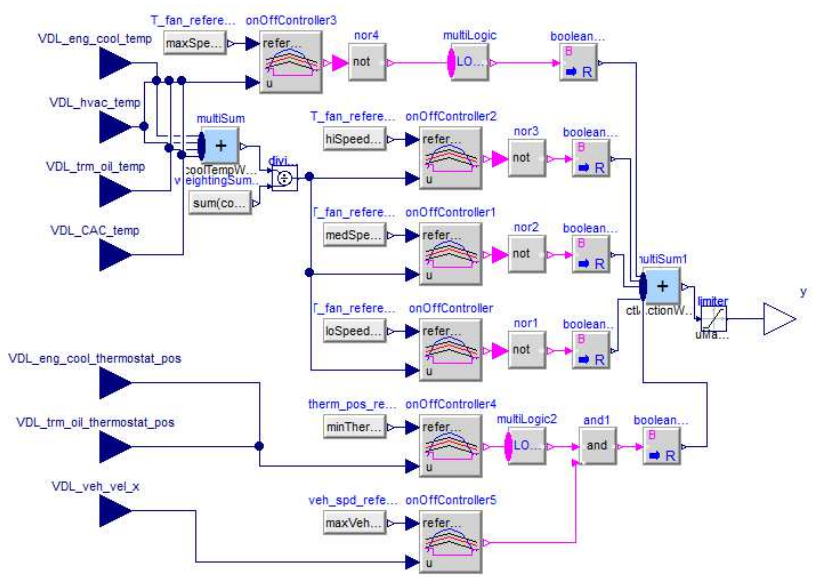

Figure 8. Simple fan controller

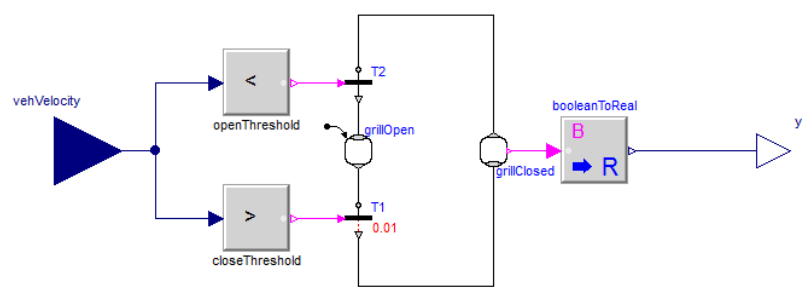

Figure 9. Passive grill shutter control

\subsection{Driver and Drive Cycles}

To support drive cycle simulations, the vehicle model shown in Figure 2 is augmented with a driver model as shown in Figure 10. The driver model provides closed loop trace following based on the vehicle longitudinal velocity. The driver model also handles the gear shifting for the automatic transmission based on a shift map. The driver model is adapted from the closed loop driver in VDL. Ambient and road conditions are also specified in the augmented driver.

Drive cycle selection is also implemented in conjunction with the vehicle model. Figure 11 shows the interface for drive cycle selection. Common and publicly available drive cycles are selectable from a drop down list. Custom drive cycles are supported via the "User Defined" option where the cycle data is implemented directly in the model or via the "File" option where the drive cycle data is read from a file.

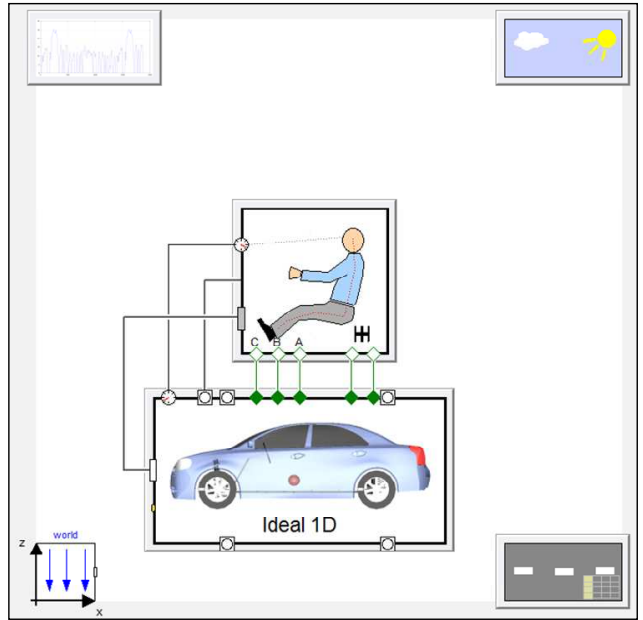

Figure 10. Assembled vehicle with driver, drive cycle, and ambient models

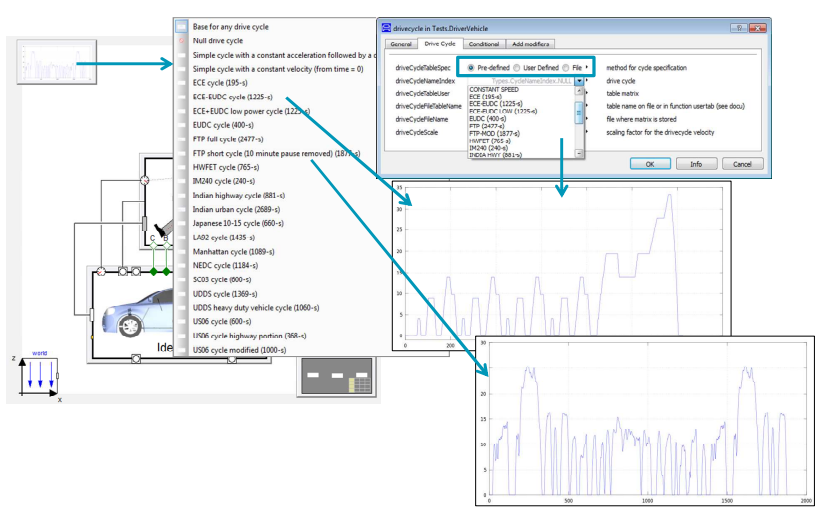

Figure 11. Drive cycle model with standard and userdefined cycle implementations

\section{Application Examples}

Using the component and subsystem models outlined in the previous section, this section details several applications and workflows using the integrated VTM model.

\subsection{Drive Cycle Simulation}

The first application simply illustrates drive cycle simulations in Dymola [10] with the models described in Section 2. The integrated VTM model is simulated on the US06 drive cycle. Selected results from that simulation are shown in Figure 12. These results illustrate both the typical drive cycle simulation results along with results of a thermal system such as coolant and oil temperatures. Even with parameterization for a demonstrator model, the results are certainly reasonable and suitable to illustrate additional workflows with the VTM model. 


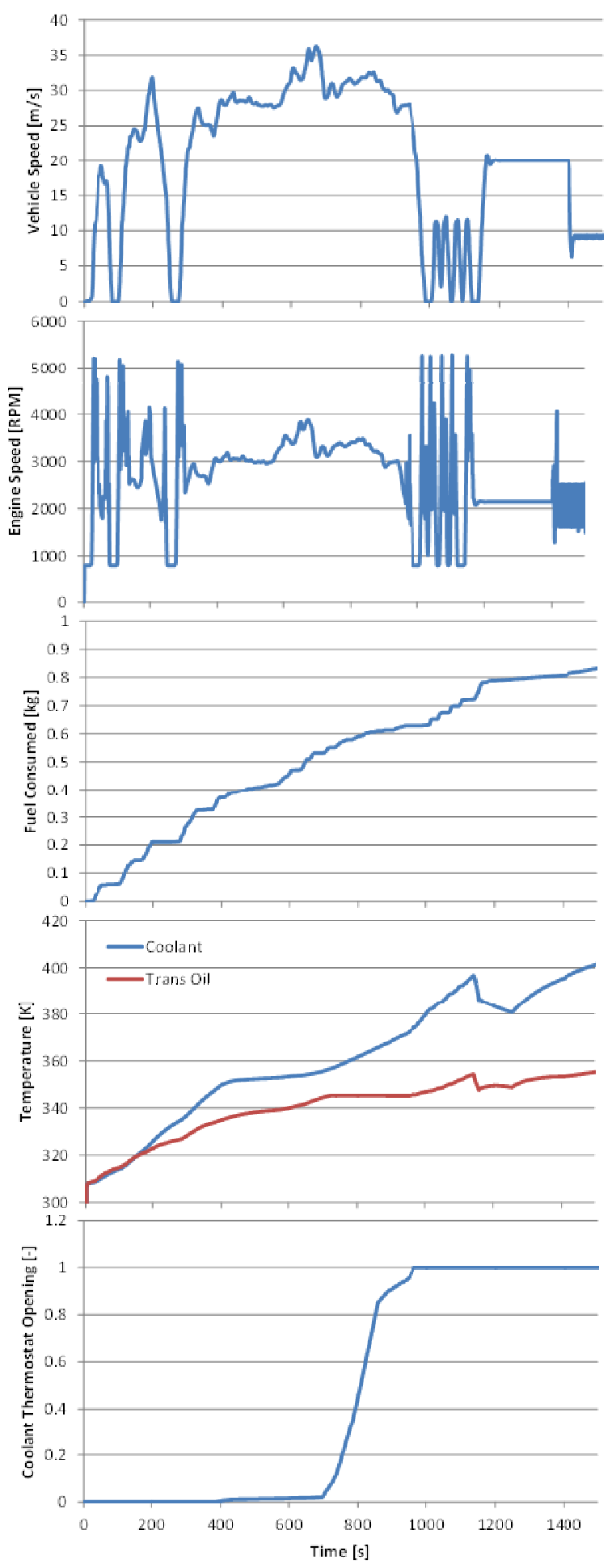

Figure 12. Simulation results on US06 drive cycle

\subsection{Controls Development and Optimization}

While Modelica can handle both controls and physical models, a common workflow is to combine Modelica-based tools for physical modeling with Simulink for controls development. This coupling between Simulink and Modelica-based tools like Dymola is especially streamlined with Functional Mock-up Interface (FMI) [6]. While Simulink is not natively FMI-compliant, Modelon provides the FMI Toolbox for MATLAB [7] that enables both import of FMUs into Simulink and export of FMUs from Simulink. This bi-directional coupling is extremely powerful and useful in that it allows both controls engineers and physical system modelers to leverage best-of-breed tools to support their work with a robust and straight-forward workflow for integration in either simulation environment.

For integration with controls in Simulink, the integrated VTM model in Figure 1 is simply modified to accept external inputs for the fan and grill commands as shown in Figure 13 to provide a portioning between the physical and control systems. A model exchange FMU is created in Dymola from this model. This FMU for the VTM model is then imported into Simulink using FMI Toolbox for MATLAB and integrated with the fan and grill controllers implemented natively in Simulink. The resulting integrated model in Simulink is shown in Figure 14.

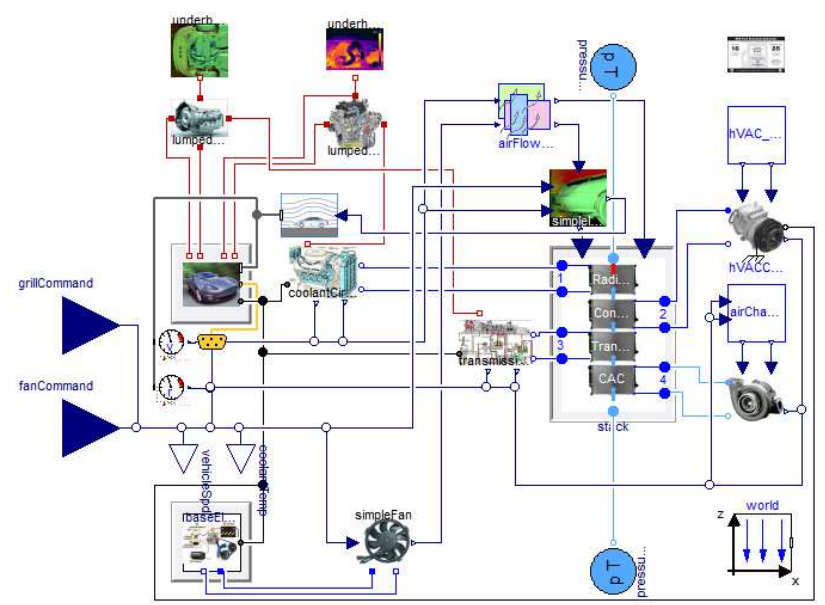

Figure 13. VTM model with external control for FMU generation

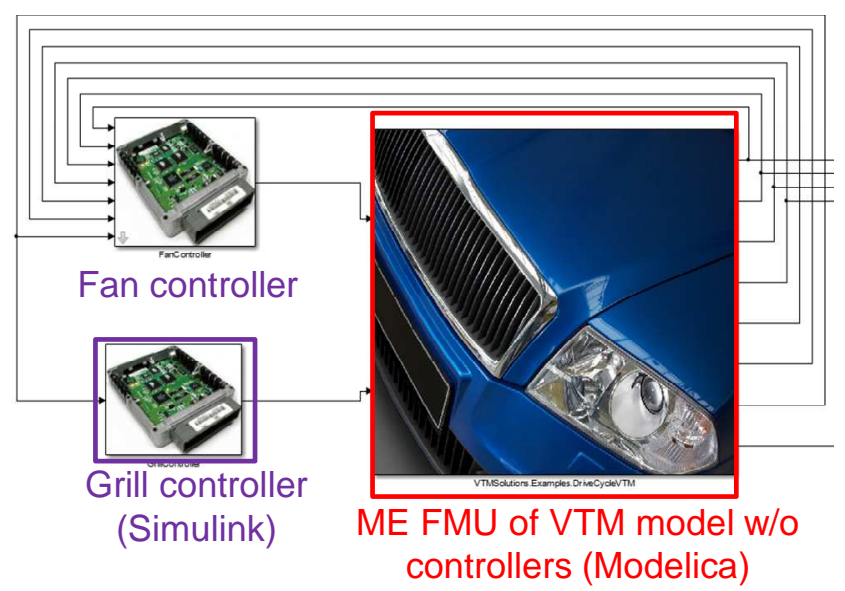

Figure 14. Integrated model in Simulink via FMU import with FMI Toolbox for MATLAB 
An active shutter control strategy was developed in Simulink to explore opportunities for improved vehicle fuel consumption. To help determine optimal settings for grill command and fan command, the DOE capabilities of FMI Toolbox for MATLAB to execute the runs and post-process results were used to run a full factorial sweep for grill and fan command at different vehicle speeds. Sample results for a vehicle speed of $120 \mathrm{kph}$ are shown in Figure 15. These DOE results can be used to identify optimal grill and fan commands that maintain desired coolant temperatures at minimum fuel consumption.

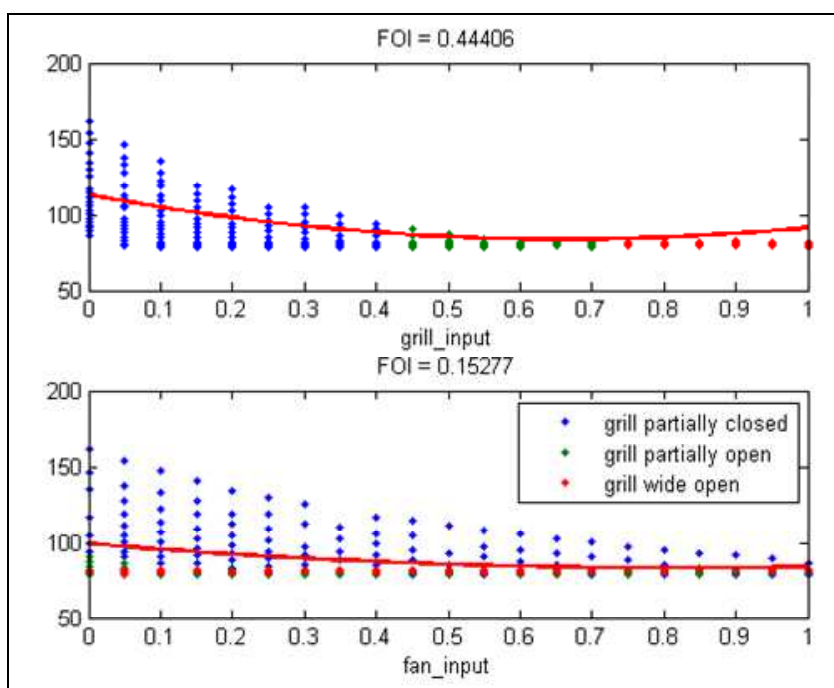

(a) Engine coolant temperature

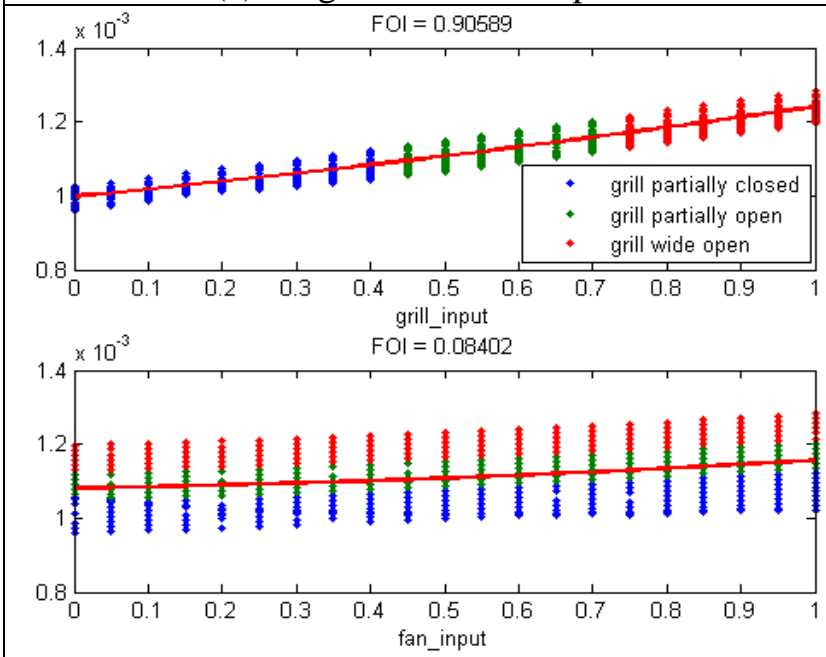

(b) Fuel flow rate

Figure 15. Results from DOE for grill and fan commands at 120kph vehicle speed

Using the DOE results, an active control strategy is implemented in Simulink. The implementation is shown in Figure 16. This strategy attempts to keep the grill closed as much as possible to reduce aerodynamic drag while maintaining target coolant and oil temperatures. The controller attempts to use op- timal grill and fan settings when operating in cooling mode.

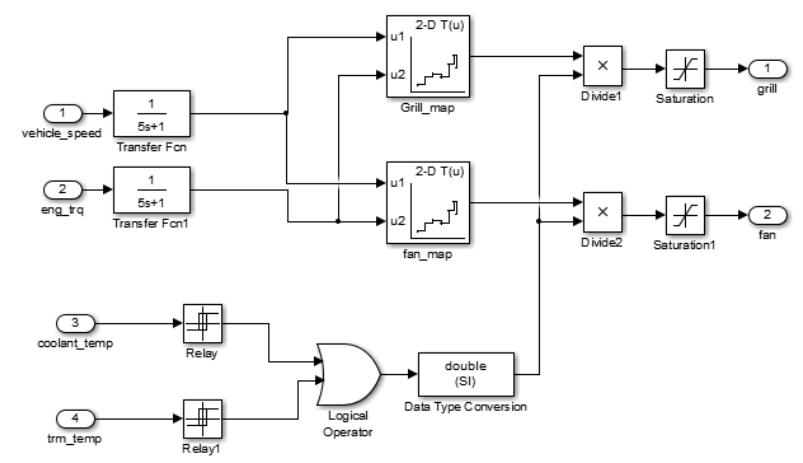

Figure 16. Active grill shutter controller

The active and passive strategies were run on a number of common drive cycles. Figure 17 shows the vehicle speed for the FTP MOD drive cycle. Figure 18 shows comparisons between the active and passive grill shutters for the FTP MOD drive cycle. For this drive cycle, the vehicle speeds are less than $15 \mathrm{~m} / \mathrm{s}$ for the majority of the cycle. The coolant temperatures between the mechanical and active shutter controllers are compared in Figure 18. The active shutter controller can be observed to speed the warm up of the coolant temperatures by at least 2 minutes in addition to maintaining the coolant temperatures close to the desired operating point of $90^{\circ} \mathrm{C}$ throughout the entire drive cycle. The grill and fan commands in the mechanical shutter controller switch between off and completely on whereas the active shutter controller makes optimal use of the cooling mechanism to both speed up the warming process and at the same time maintaining temperatures close to the desired operating point. When compared at equivalent coolant temperatures, the optimal controller is expected to have slightly better fuel consumption (typical benefits of grill shutters are in the range of $0.5-2 \%$ depending on the vehicle and cycle). Of course, these results are highly dependent on the power consumption of the fan and the tradeoff between fan power and recovered aero drag and highlight the need for an analytic model to comprehend these complex tradeoffs.

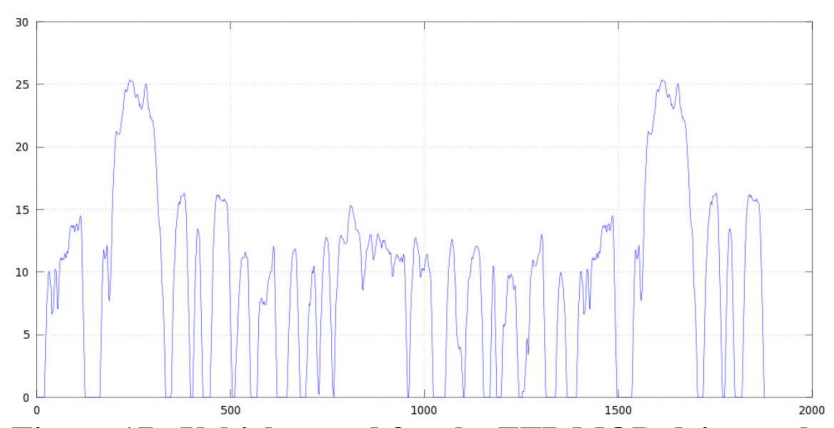

Figure 17. Vehicle speed for the FTP MOD drive cycle 


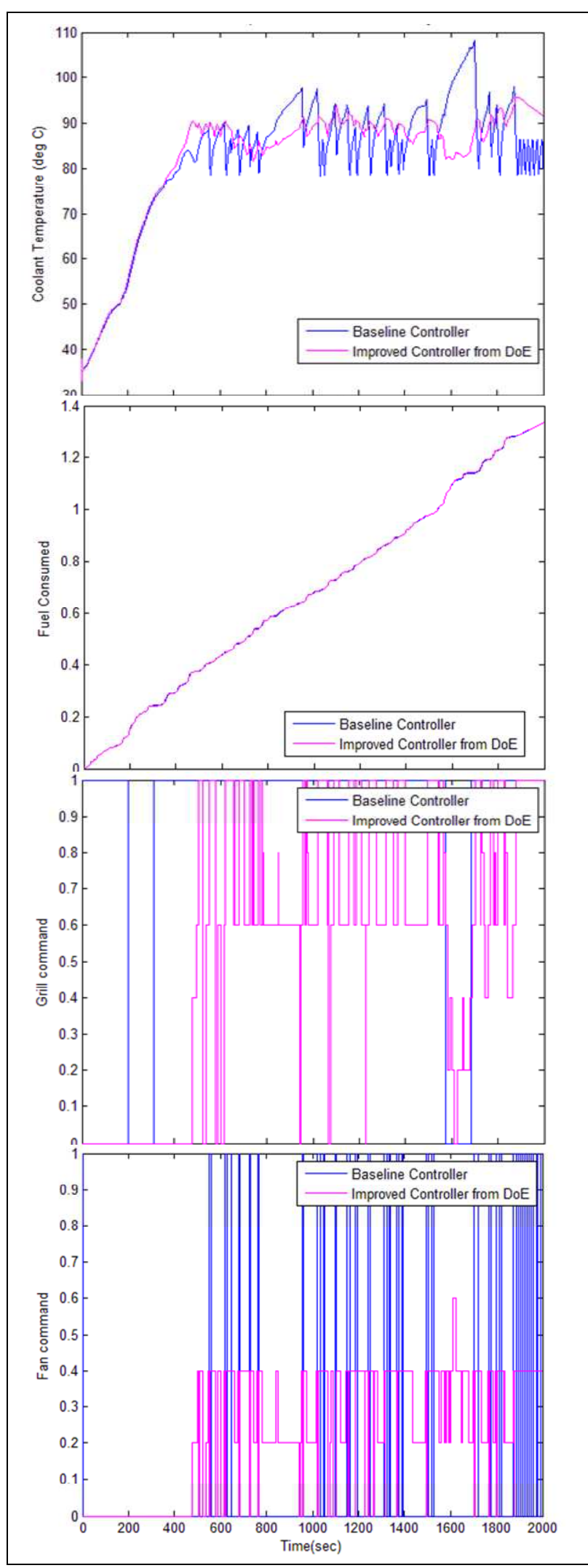

Figure 18. Comparisons between active and passive grill shutters on FTP MOD cycle

\subsection{Batch Simulations and Robustness}

The integrated VTM model can be simulated from Excel using FMI Add-in for Excel to support batch simulations and robustness applications. To support this workflow, a co-simulation FMU is created and then imported into FMI Add-in for Excel. Experiment sheets allow users to change parameters, provide external inputs, apply boundary conditions, simulate the model, and post-process the results. The simulations are automatically run in parallel distributed across the local CPU cores. The use of FMI for model deployment outside of the model development environment is providing additional value from a standards-based workflow.

A sample experiment sheet for the VTM model is shown in Figure 19 to run the model over various drive cycles. Coolant temperature results from a batch simulation at a range of vehicle speeds are shown in Figure 20. Using the scripting API provided with FMI Add-in for Excel, Monte Carlo simulations for robustness applications are enabled. Figure 21 shows the Monte Carlo experiment sheet created by the script and results from simulations over a distribution of heat exchanger effectiveness multipliers and airflow distribution multipliers.

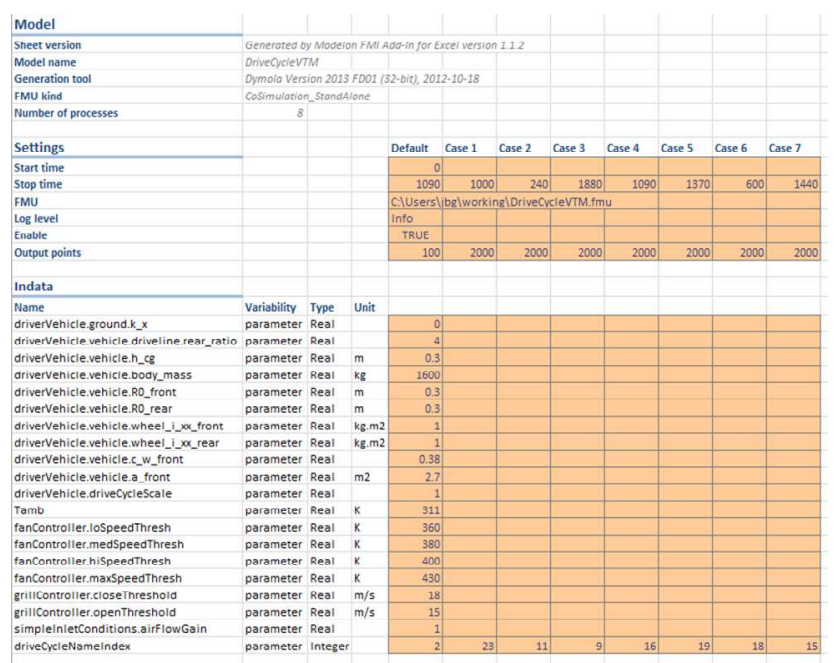

Figure 19. Experiment sheet in Excel to run model over different drive cycles

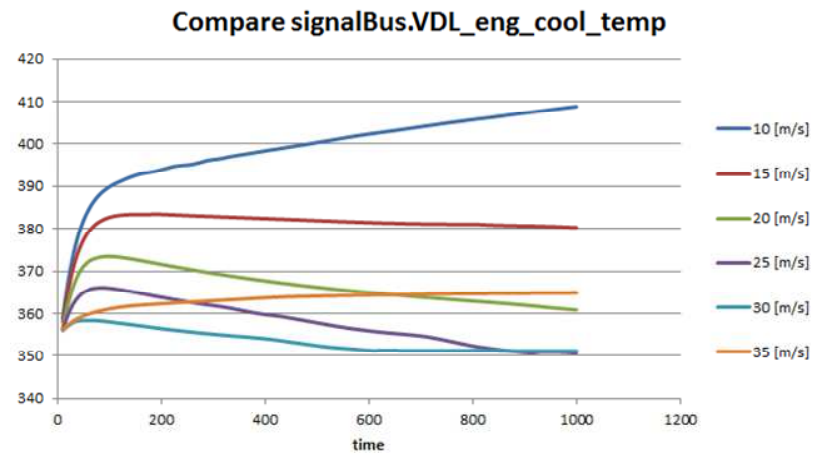

Figure 20. Batch simulation showing coolant temperature over a range of vehicle speeds 


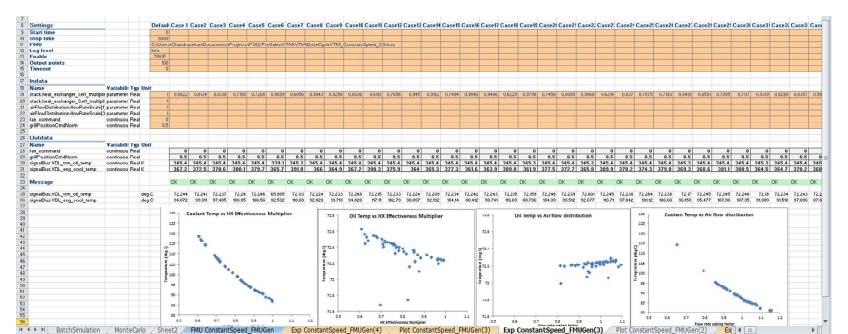

Figure 21. Monte Carlo simulations for heat exchanger effectiveness and stack airflow

\subsection{Heat Exchanger Non-Uniformity}

Heat exchanger performance is a critical factor in VTM applications. While convenient to simulate with uniform velocity and temperature inputs, actual conditions typically include non-uniformity. Thus, assessing heat exchanger performance under nonuniform conditions is critical. Heat Exchanger Library [5] provides both uniform and non-uniform input sources. Figure 22 shows a test model that can be configured for either uniform or non-uniform inputs. Non-uniform inputs would typically be provided from a CFD tool and thus represent a 1D-3D coupling between CFD and the discretized 1D approach in the models in Heat Exchanger Library. Similar coupling with the Air Conditioning Library [12] for evaluating idle air recirculation has been published [13].

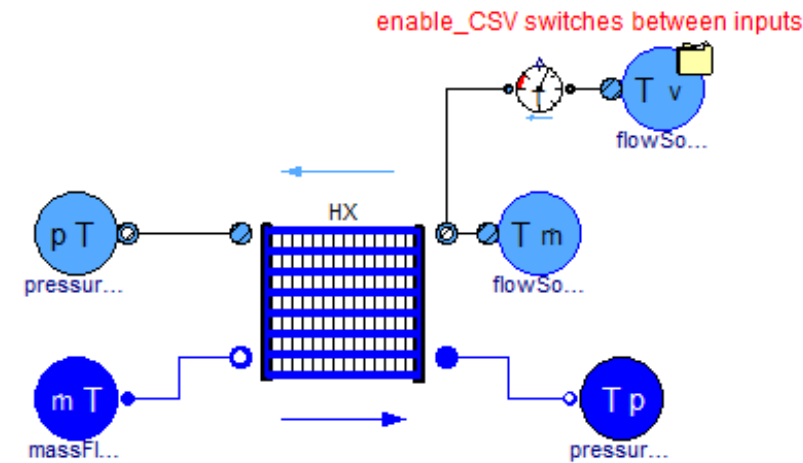

Figure 22. Heat exchanger test with option for uniform or non-uniform inputs

To verify the model, published distributions [11] were simulated and compared with analytic results from the publication for heat exchanger nonuniformity, defined as the ratio of heat transfer for non-uniform inputs to heat transfer with averaged uniform inputs from the non-uniform distribution:

$$
\text { NonUniformityFactor }=\frac{Q_{\text {non-uniform }}}{Q_{\text {uniform avg }}}
$$

The study was performed with a model from Heat Exchanger Library calibrated to bench data. The model was then run over the published distributions in [11] for a range of heat capacity ratios with the external air as the minimum heat capacity fluid and non-uniformity results compared with published values. Figure 23 shows a sample distribution. The comparisons between the HXL simulation and the published results are shown in Table 1. Note that the results from the paper were extracted from graphs in [11]. The simulations in [11] were also run over a large range of NTU values (0-100) and it was difficult to extract values at the NTU for this cooler (roughly 1-2). Thus, the values for NTU=5 and NTU approaching zero were extracted for comparison with the model and shown in the table. The model accurately captures both the trend and magnitude of the non-uniformity.

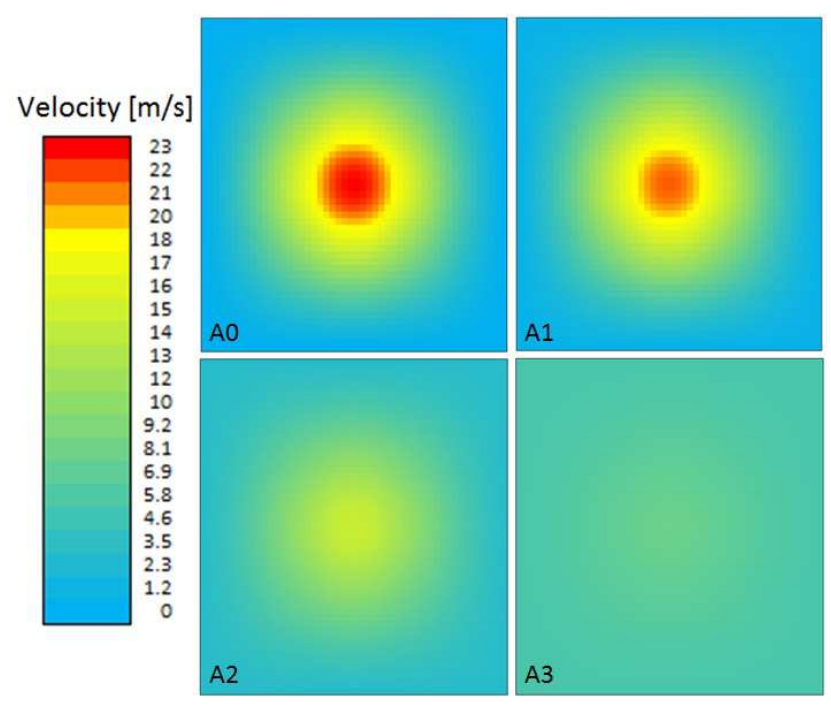

Figure 23. Velocity distributions from patterns in [11]

Table 1. Non-uniformity comparison between model and published results [11]

\begin{tabular}{|c|c|c|c|c|}
\hline \multirow{3}{*}{ Distribution } & \multirow{3}{*}{$\mathrm{Cmin} / \mathrm{Cmax}$} & \multirow{2}{*}{$\begin{array}{l}\text { HXLSimulation } \\
\text { Non-Uniformity }\end{array}$} & \multicolumn{2}{|c|}{ Ranganayakulu Paper } \\
\hline & & & $\mathrm{NTU}=0$ & $\mathrm{NTU}=5$ \\
\hline & & Interpolated & Non-Uniformity & Non-Uniformity \\
\hline A0 & 0.2 & 0.820 & 0.818 & 0.846 \\
\hline A1 & 0.2 & 0.859 & 0.856 & 0.874 \\
\hline A2 & 0.2 & 0.959 & 0.950 & 0.958 \\
\hline A3 & 0.2 & 0.998 & 0.991 & 0.993 \\
\hline AO & 0.4 & 0.815 & 0.769 & 0.850 \\
\hline A1 & 0.4 & 0.860 & 0.831 & 0.900 \\
\hline $\mathrm{A} 2$ & 0.4 & 0.961 & 0.948 & 0.956 \\
\hline A3 & 0.4 & 0.998 & 0.993 & 0.995 \\
\hline AO & 0.6 & 0.814 & 0.750 & 0.821 \\
\hline A1 & 0.6 & 0.862 & 0.810 & 0.862 \\
\hline $\mathrm{A} 2$ & 0.6 & 0.962 & 0.946 & 0.949 \\
\hline A3 & 0.6 & 0.998 & 0.991 & 0.991 \\
\hline AO & 0.8 & 0.815 & 0.811 & 0.809 \\
\hline A1 & 0.8 & 0.864 & 0.844 & 0.840 \\
\hline A2 & 0.8 & 0.963 & 0.946 & 0.938 \\
\hline A3 & 0.8 & 0.998 & 0.989 & 0.989 \\
\hline AO & 1 & 0.816 & 0.810 & 0.808 \\
\hline A1 & 1 & 0.866 & 0.845 & 0.842 \\
\hline$A 2$ & 1 & 0.964 & 0.971 & 0.945 \\
\hline A3 & 1 & 0.998 & 0.999 & 0.998 \\
\hline
\end{tabular}




\section{Conclusions}

A coordinated suite of Modelica libraries for vehicle thermal management applications have been used in the implementation of an integrated VTM model with combined vehicle fuel and thermal effects, including the key physical and control models. The demonstrator VTM model is implemented using the Vehicle Dynamics Library, Liquid Cooling Library, and Heat Exchanger Library from Modelon. Several application examples focused on vehicle thermal management have been detailed. These application examples include drive cycle simulations, controller development and optimization, batch simulations and robustness applications, and 1D-3D coupling for heat exchanger performance. These application examples demonstrate the use of sophisticated model libraries to enable the multi-domain approach needed for vehicle thermal management applications. The application examples also illustrate the use of FMI to couple the VTM model with controls in Simulink and for use in robustness application in Excel. Given the importance of model deployment outside of traditional CAE environments to support model-based systems engineering, workflow aspects via FMI are highlighted.

\section{References}

[1] Bouvy, et al., "Holistic Vehicle Simulation using Modelica - An Application on Thermal Management and Operation Strategy for Electrified Vehicles", Proceedings of $9^{\text {th }}$ International Modelica Conference, pp. 263270, 2012.

[2] King, Jenny., "Shooting the breeze about active grille shutters: What do they do?", http://cars.chicagotribune.com/fuel-

efficient/news/chi-active-grille-shutters20130613, June 13, 2013.

[3] Andreasson, J., "The Vehicle Dynamics Library: New Concepts and New Fields of Application", Proceedings of $8^{\text {th }}$ International Modelica Conference, 2011.

[4] Modelon, "Liquid Cooling Library", Version 1.1 , 2013. http://www.modelon.com/products/modelicalibraries/liquid-cooling-library/

[5] Modelon, "Heat Exchanger Library", Version $1.0, \quad 2013$ http://www.modelon.com/products/modelicalibraries/heat-exchanger-library/
[6] MODELISAR, "Functional Mock-up Interface for Model Exchange", Version 1.0, 2010.

[7] Modelon, "FMI Toolbox for MATLAB", Version $1.7,2013$. http://www.modelon.com/products/fmitoolbox-for-matlab/.

[8] Modelon, "FMI Add-in for Excel", Version 1.2.1, 2013. http://www.modelon.com/products/fmi-addin-for-excel/

[9] Griffin, J., Batteh, J., and Andreasson, J., "Modeling Vehicle Drivability with Modelica and the Vehicle Dynamics Library", Proceedings of $9^{\text {th }}$ International Modelica Conference, pp. 599-608, 2012.

[10] Dassault Systemes, "Dymola 2013 FD01", 2012.

[11] Ranganayakulu, CH., Seetharamu, K.N., and Sreevatsan, K.V., "The Effects of Inlet Fluid Flow Nonuniformity on Thermal Performance and Pressure Drops in Crossflow Plate-Fin Compact Heat Exchangers", International Journal of Heat and Mass Transfer, Vo. 40, No. 1, pp. 27-38, 1997.

[12] Eborn, et al., "AirConditioning - a Modelica Library for Dynamic Simulation of AC Systems, Proceedings of $4^{\text {th }}$ International Modelica Conference, pp. 185-192, 2005.

[13] Wang, et al., "Integrated Thermal Management Simulations: Evaluating the Effect of Underhood Recirculating Airflows on ACSystem Performance, Proceedings of $7^{\text {th }}$ International Modelica Conference, pp. 413422, 2009. 